als Vorbereitung aufs politische Examen wurde sichtbar bei Lenin, der 1914 die proletarische Revolution als den Schlußpunkt des beginnenden I. Weltkrieges voraussagte und ein Jahr darauf sein eingehendes kritisches Studium der Hegelschen Philosophie vornahm. Hegelsche Dialektik, die "Algebra der Revolution" (Herzen), bewahrte und bewahrt ihre Affinität zur Politik über den Umkreis bürgerlicher Politik hinaus.

Doch das Bündnis von Philosophie und Politik, das Hegel schloß, lebt im Marxismus-Leninismus sehr verwandelt fort. In der Hegelschen Form läßt es sich inzwischen auch die antiklassische Bourgeoisie unserer Tage gefallen. Sie kann ihrer eigenen Geschichte sogar den Tort antun, daß einzelne Strömungen durchaus bürgerlichen kritischen Zeitverständnisses das Spiel mit klassischen Denkformen zur Mode machen und findet es pikant, da $B$ dabei auch ins Marxsche Feld elegant und flüchtig mit hineingespielt wird. Die große zeitgemäße Erneuerung der klassischbürgerlichen Verknüpfung von Philosophie und Politik, die schon vor 100 Jahren fällig war und vollzogen wurde, finden wir dabei ignoriert oder als Abbiegung eines sog. humanistisch-praktischen Ansatzes von Marx auf tote Gleise denunziert, die zu seelenlosem Determinismus und zu dogmatischer Parteipolitik führten. Die Evolution der Hegelschen Auffassung von der geschichtlich aktiven Rolle der Philosophie gibt AufschluB über die Schwächen des Praxisverständnisses der bürgerlichen Ideologie.

\title{
II. Spekulation und Gewalt
}

Die erste Ausbildungsstufe der politischen Auffassungen Hegels (etwa 1790-1798) zeichnete sich durch das Fehlen einer konkreten historischen und politischen Konzeption aus. Hegel huldigte dem unbestimmten Ideal einer sittlichen Gemeinschaft des Volkes. Es war ein von Rousseau - Hegels Lieblingsschriftsteller in seiner Tübinger Studentenzeit - beeinflußtes demokratisches Ideal, das sich Hegel aber bezeichnenderweise nicht in Form von politischen Schriften, sondern durch historische, vor allem 
religionshistorische Studien vergegenwärtigte. In einem sehr allgemeinen Sinne ist es wohl zutreffend, daß Hegel damals ein republikanisches Staatsideal besessen habe. Aber es war darum gerade keine politische Auffassung des Staates. In jenem allgemeinen Sinne hat Hegel das Ideal der Demokratie bis an sein Lebensende festgehalten (die Reformbill-Schrift gibt es noch zu erkennen) - aber von seinen politischen Auffassungen wohl gesondert.

Der Standpunkt des unbeschränkten, nicht-positiven sittlichen Volkslebens gab als Standpunkt des gegen die Wirklichkeit gerichteten Ideals dem philosophischen Bewußtsein allerdings eine aktive, Wirklichkeit verändernde, neue Wirklichkeit erzeugende Stellung. Hegel studierte damals, 1794/95 in Bern, nicht zufällig Kants praktische Philosophie, Fichtes Wissenschaftslehre und nannte Fichte mit Hölderlin einen "Titanen, der für die Menschheit kämpfe“. 1 Die Transzendentalphilosophie sei Beweis, daß der Nimbus um die Häupter der Despoten und Götter der Erde vor der steigenden Selbstachtung der Menschen verschwinde. Und nun über die Rolle des philosophischen Bewußtseins: „Die Philosophen beweisen diese Würde, die Völker werden sie fühlen lernen, und ihre in den Staub erniedrigten Rechte nicht fodern, sondern selbst wieder annehmen, - sich aneignen ... Mit Verbreitung der Ideen, wie Alles sein soll, wird die Indolenz der gesetzten Leute ewig alles zu nehmen, wie es ist, verschwinden." 2 Es ist hier klar umrissen: Philosophie ist Kritik, sie setzt neue Wirklichkeit. Der außerhalb der ideellen Kritik stehende reale Prozeß der Setzung wird aber nicht reflektiert. Die Praxis der kritischen Vernunft bleibt Vernunftpraxis; Realpraxis fällt außerhalb der kritischen Vernunft.

Auf der zweiten, nun erst eigentlichen Stufe der Ausbildung von politischen Auffassungen (etwa 1798-1817) ging Hegel daran, die idealische Unbestimmtheit seiner politischen Gedanken zu überwinden. Er hatte sich durch religionsgeschichtliche, ökonomische und allgemeine historische Studien davon überzeugt, daß das Ideal demokra-

1 Hegel an Schelling, Jan. 1795

2 Hegel an Schelling, 16. 4. 1795 
tischer Sittlichkeit in der ihm vorliegenden Gesellschaft mit ihren starr gesonderten Ständen, mit durchdringendem bürgerlichem Privateigentum und Privatinteresse, nicht realisierbar sei. Hegel suchte - mit der inzwischen erworbenen außerordentlichen Fähigkeit historischen Denkens nach einer politischen Vermittlung von progressiver Vernunftforderung und der gegebenen politischen Situation Deutschlands. Der Gang der Französischen Revolution bildete für Hegels Denken wie für die Wandlung der bürgerlichen Ideologie in Deutschland überhaupt die große Triebkraft: die Bewegung von der konstitutionellen Monarchie der girondistischen Verfassung (1791) über das Zwischenspiel der 1793 von Robespierre verkündeten fast idealen demokratischen, nicht zufällig aber sofort suspendierten Verfassung zur nachdrücklichen Wiederkehr der monarchischen Konstitution nach dem 18. Brumaire 1799.

Hegels politisches Denken ist von der Erkenntnis bestimmt, daß der Übergang von der feudalen zur kapitalistischen Gesellschaft das historische Gesetz ist, das die Epoche bestimmt. Hegel faßte diese Einsicht in einer Entwicklungsphase des Kapitalismus, in der die ihn auszeichnende Klassenspaltung der Gesellschaft im Land der Revolution und erst recht in Deutschland noch nicht ausgebildet war. Er verwarf daher die Repräsentativverfassung der liberalen bürgerlichen Demokratie und entwickelte den ständisch organisierten Staat der konstitutionellen Monarchie als den Staat der aus dem Feudalismus herausgetretenen Gesellschaft. Dieser Staatsbegriff ist in der Landständeschrift (1817) und darauf wieder in der Rechtsphilosophie (1821) umfassend dargestellt.

Die bezeichneten Schicksale der Französischen Revolution waren für den mit der Jahrhundertwende eintretenden Strukturwandel des klassischen bürgerlichen Denkens in Deutschland von bestimmendem EinfluB. Durch sie wurde der schon seit den siebziger Jahren des 18. Jahrhunderts auf vorwiegend naturwissenschaftlich-pantheistischer Grundlage vorbereitete objektive Idealismus zur herrschenden Richtung des philosophischen Denkens. Der Standpunkt der Vernunftforderung war schwer erschüttert. Ein Teil der Enthusiasten, die wie Fr. Schlegel noch 1799 die Revolu- 
tion als eines der Ereignisse des Jahrhunderts gefeiert hatten, wandten sich deprimiert vom bösen Zeitgeist ab. Mit der romantischen Reaktion auf die französische Revolution setzte die Krise der klassischen bürgerlichen Ideologie ein. Diese Krise war von dem im bürgerlichen Denken - wie wir das oben an Hegel kurz illustrierten - nicht reflektierten Problem der Realisierung der Vernunftforderungen herbeigeführt worden. Das Problem hatte sich im Gang der französischen revolutionären Praxis als unabweisbar gezeigt. Doch die Denker, die an der Vernunftforderung einer neuen Gesellschaft, auch der freien Monarchie, wie Hegel die konstitutionelle Monarchie nannte, festhielten, standen ebenfalls unterm Eindruck der Erschütterung des ursprünglichen Vernunftideals durch das welthistorische Unternehmen seiner Realisierung jenseits des Rheins. Das progressive Denken mußte sich vor dem Problem der Verwirklichung der Vernunft bewähren. Nun trat Hegel an die Spitze des klassischen bürgerlichen Denkens in Deutschland.

Die Erschütterung des Vernunftideals war bei ihm in doppelter Hinsicht erfolgt. Er hatte, wie gesagt, die prinzipielle Kluft zwischen sittlich-demokratischem Ideal und gegebener Gesellschaft, die Unrealisierbarkeit des Ideals erkannt und wandte sich nun mit der ganzen Wucht seines aus langer, oft qualvoller innerer Arbeit an den Tag tretenden überragenden Geistes gegen die Philosophie des seinsollenden Ideals. Doch Hegel war mit seiner Erkenntnis zugleich auf das Problem der gegenständlichen Realisierung gestoßen. Eine die neue Wirklichkeit konzipierende Philosophie hatte bestanden. Der reale ProzeB ihrer Verwirklichung in Frankreich blieb ihm weitgehend unverständlich, und er lehnte ihn ab. In Deutschland war der kolossale Anlauf, den die Ideologie des seinsollenden Ideals genommen hatte, ohne jedes praktische Resultat geblieben. Hegel stand in seiner Frankfurter Zeit vor einem Widerspruch, der sein ganzes, nach festem Grund für die Wirklichkeit der bürgerlichen Vernunftforderungen suchendes Denken erschütterte. Er mußte von der Berechtigung revolutionärer Umgestaltungen Deutschlands überzeugt sein. Das bestehende Deutsche Reich, die elende, fast groteske 
Ruine der Feudalgesellschaft in Deutschland, hatte, wie Hegel später zu unterscheiden pflegte, wohl Existenz, aber keine Wirklichkeit für sich. (Dieses Thema steht im Mittelpunkt der Schrift über die Reichsverfassung, 1799-1802.) Zugleich sah er, da $B$ die schlechte Wirklichkeit gegen die bloße Einsicht der Philosophie standhielt und niemand daran ging, die von der Theorie bewiesene „Würde der Menschheit" aufzunehmen. Hegel gelangte vor die Grenze des bürgerlichen ideologischen Praxisverständnisses.

Hegel notierte in der Verfassungsschrift über die reale Erneuerung des Deutschen Reiches, die er als den politischen Utbergang von der feudalen zur bürgerlichen Gesellschaft in Deutschland verstand: „... der Begriff und die Einsicht der Notwendigkeit (ist) viel zu schwach ..., um aufs Handeln selbst zu wirken; der Begriff und Einsicht führt etwas so Mißtrauisches gegen sich mit, da $B$ er durch die Gewalt gerechtfertigt werden $\mathrm{muB}$, dann unterwirft sich ihm der Mensch" (S. 111). Wir sehen, Hegel formuliert die ernste Erfahrung jeder „kritischen Theorie“, auch sog. „Philosophie der Praxis“, wenn diese wirklich will, was sie weiß. Die sich um die zitierte Problematik konzentrierenden Aspekte der Verfassungsschrift sind das philosophisch bedeutsame Moment dieses politischen Manuskripts. Das politische Werk läßt die umfassenden geschichtlichen Probleme, zu deren Lösung Hegel seine Philosophie des konkreten, d. i. des idealen und realen, Begriffs formulierte, deutlich werden. Hat Hegel das von ihm aufgeworfene Problem realer Praxis gelöst? Er hat es nach dessen Formulierung im komplexen historischen Zusammenhang nochmals in abstrakter analytischer Form aufgenommen. Im „System der Sittlichkeit“ (1801/02) sowie in den beiden ,Jenenser Realphilosophien" (1803-1806) untersuchte er das Problem der Vergegenständlichung im einfachen ArbeitsprozeB. Es zeigt Hegels Genie, da $B$ er zu dieser einzig echten Keimzelle der Grundprobleme der Philosophie vordrang. Er gelangte da zu Resultaten, die hinsichtlich des gegenständlichen Charakters der Praxis, hinsichtlich des Úbergangs von Subjektivität in Objektivität über die Feststellungen der Verfassungsschrift hinausgingen. Aber doch nur um den Preis, da $\beta$ die Fragestellung vom konkreten Problem historischer 
Praxis, revolutionärer geschichtlicher Umgestaltung abgezogen ist.

Hegel weist in der politischen Schrift auf einen praktischen politischen Ausweg: Nur die Gewalt eines Eroberers (Napoleons) kann das Deutsche Reich erneuern (S. 110). Aber wie war diese reale Gewalt der Revolution aus der Idee der revolutionären Veränderung entstanden? Hegel bezeichnet diese Frage ausdrücklich als unlösbar. Am Ende eines Entwurfs zur Einleitung, der die tieferen philosophischen und methodischen Probleme ausführt, die Hegel bei der Abfassung der politischen und historischen Studie bewegten, heißt es: Das Gefühl des Widerspruchs der Vernunft mit der bestehenden Wirklichkeit ist das Bedürfnis, da $B$ er gehoben werde. Das Bewußtsein des Widerspruchs, das sich „an den Bewegungen ganzer Völker (d. i. der französischen Revolution und der Revolutionskriege - G. I.), an der Darstellung der Natur und des Schicksals durch Dichter" (d. i. der klassischen deutschen Literatur - G. I.) nährt (S. 118), erhöht das Leiden der Menschheit, wenn die reale Veränderung ausbleibt. Eine wohl auch für die persönliche Geistesgeschichte Hegels aufschlußreiche Stelle. Wie soll aber die schlechte Wirklichkeit beseitigt werden? Hegel antwortet: „Das beschränkte Leben als Macht kann nur dann von Besserem feindlich mit Macht angegriffen werden, wenn dieses auch zur Macht geworden ist ... Als Besondres gegen Besondres ist die Natur in ihrem wirklichen Leben, der einzige Angriff oder Widerlegung des schlechtern Lebens, und eine solche kann nicht Gegenstand einer absichtlichen Tätigkeit sein" (ebd.). In den letzten Worten hat Hegel seine Überzeugung ausgesprochen, da $B$ theoretisch bewußte revolutionäre Praxis unmöglich sei.

Es ist aus den politischen Texten und Briefen Hegels klar zu ersehen, warum die Philosophie der Vernunft das Problem rationaler gesellschaftlicher Praxis abweist und so tatsächlich einen irrationalen Rest im Wesen des Wirklichen konstituiert. Wir wollen darauf im späteren $\mathrm{Zu}$ sammenhang mit der Interpretation einiger politischer $\mathrm{Hal}$ tungen Hegels eingehen. Das irrationale Moment im klassischen Vernunftsidealismus hatte weitreichende Folgen für Hegels politische Konzeption wie für seine philosophische 
Systemarbeit überhaupt. Zunächst ist der Widerspruch zu bemerken, der im theoretischen Reifeprozeß der politischen Auffassungen Hegels auftrat. Indem er seine Gesellschaftslehre konkretisłerte, sie vom antikisierenden Ideal der Sittlichkeit zu einer Lehre von der existierenden Wirklichkeit und für die gegebene Wirklichkeit erhob, wurde er sich der Unmöglichkeit bewußt, rationale geschichtliche Praxis als gegenständlichen Vorgang theoretisch zu fassen. Geschichte wurde bei Hegel konkreter ProzeB, Reichtum der gesellschaftlichen Formen; dialektisch große Anschauung der Wirklichkeit gestaltete sich. Aber Geschichte wurde nicht praktischer Vorgang, sie wurde nicht unterm Gesichtspunkt „absichtlicher Tätigkeit“ verstanden. Hegels Übergang von der subjektiven formalen zur objektiven, wirklichen Inhalt besitzenden Vernunft blieb dadurch unvollendet. Das ist, wenn man so will, der unüberwundene transzendentale Idealismus im System Hegels.

Die Realisierung der Vernunft erscheint bei Hegel stets nur als vollzogene, nie als zu vollziehende. Darum muß auch die Untersuchung der jeweils schon vollzogenen oder noch zu vollziehenden Utbereinstimmung von kritischer Idee und existierender Wirklichkeit sehr ungenau werden. Im Nebel unbegriffener Realisierung breitet sich die Macht der widerständigen schlechten Wirklichkeit aus. Ohne den zentralen Begriff rationaler Praxis als eines gegenständlichen Vorgangs von tatsächlicher ,Gewalt" ist keine Ưberprüfung des jeweiligen Erfüllungsstandes der Einheit von kritischer Theorie und Realität möglich.

Hegel sieht, daß die kritische Theorie zu gegenständlicher gesellschaftlicher Praxis übergehen muß, aber er weist diese aus dem Bereich des theoretisch Erfaßbaren hinaus. Praxis wird zum Unding absichtsloser Tätigkeit. Sowenig Hegel den Übergang der kritischen Idee in die Wirklichkeit tatsächlich begreift, sowenig kann er beim Gedanken der andauernden Entzweiung von Idee und Realität stehenbleiben. Er ist daher gezwungen, entweder das politische Programm der Erneuerung der Gesellschaft und das philosophische Programm einer sich verwirklichenden absoluten Vernunft aufzugeben oder die Versöhnung der kritischen Idee auch mit der vorhandenen Wirklichkeit herbeizu- 
führen. Es ist bekannt, da $B$ Hegel konsequent den zweiten Weg beschritten hat. Die existierende Wirklichkeit, zunehmend mürrisch und mit abnehmender Genauigkeit besehen, mußte dann eine idealische Erhöhung erfahren. Die Konstruktionen der spekulativen Vernunft wurden die Rose im Kreuz der Gegenwart, wie Hegel sagte. ${ }^{1}$ Nicht mehr war dem Freiheitsbaume der Tübinger Maienzeit entsprossen. Der idealische Glanz von Ubermut, den Hegel an Fichte so geschmäht hatte, er kehrte wieder als spekulative Idealisierung der Resignation. Die Grundforderung nach Verwirklichung der Vernunft wurde erfüllt als Einbildung der Vernunft ins vorhandene Wirkliche. (Goethes Uberwindung des Sturm und Drang durch den klassischen Realismus des objektiven Gehalts der Wirklichkeit zeigte den gleichen Verlust im Fortschreiten. Der Reifungsprozeß $B$ des genialischen Tasso zur Erkenntnis und Anerkenntnis obrigkeitlicher Lebensreife steht im gleichen $Z$ wielicht von Realitätsgewinn und Kompromiß und ist das ästhetische Gegenstück zum Verschwimmen von Realisierung und Versöhnung in Hegels Idealismus.)

Wir stellten oben fest, da $\beta$ ohne den Begriff rationaler Praxis als eines objektiven Vorgangs keine Uberprüfung der Beziehungen von kritischer Theorie und Wirklichkeit möglich ist. Die theoretische Schwäche muB in den spezifischen politischen Auffassungen und Verhaltensweisen zu Unbestimmtheit, Schwankungen, Ungenauigkeiten führen. Neben generöser, welthistorisch dimensionierter Großzügigkeit muß kleinliche Beschränkung auf isolierte Tatsachen einhergehen. Der Zwiespalt von völliger Unzufriedenheit mit dem Erreichten, also von Resignation einerseits und Akkomodation ans undurchdringlich Gegebene andererseits, ist dann der Satansengel, der den verlassenen Denker mit Fäusten schlägt. Die dritte Periode der politischen Auffassungen Hegels (1817-1831) ist vor allem durch diese Widersprüche, deren theoretischen Ursprung wir zu zeigen versuchten, gekennzeichnet. In ihr kommt in der politischen wie in der philosophischen Konzeption nichts mehr hinzu. Aber die prinzipiellen Wurzeln der politischen Theorie zei-

1 In der Vorrede zur Rechtsphilosophie, Berlin 1956, S. 16 
gen ihre Konsequenzen. Spekulation und Politik müssen sich bewähren.

Hegel ist in allen seinen auf die Verfassungsschrift folgenden Werken dem Versuch der Überprüfung des Verhältnisses von kritischer politischer Idee und existierender politischer Wirklichkeit konsequent aus dem Wege gegangen. In einer Diskussion mit Niethammer über die Wege des Fortschritts nach der Niederlage Napoleons ließ Hegel diesen wunden Punkt seiner spekulativen Rechtfertigung des Fortschrittsgedankens auf bezeichnende Weise deutlich werden (Hegel an Niethammer, 5. 7. 1816).

Letzten Endes liegt in Hegels Ausweichen vor einer Utberprüfung der Beziehung von politischer Theorie und realer politischer Existenz nur wieder ein Unverständnis für den gegenständlich-praktischen Realisierungsproze $B$ der Theorie (allerdings auch die Abstraktheit der politischen Theorie der konstitutionellen Monarchie als des Staates der „neueren Zeit").

Sowohl die Landständeschrift wie die Rechtsphilosophie weichen Fragestellungen solcher Art aus. Hegel läßt nie ein Wort über die Beziehung des preußischen Staates zwischen Karlsbader Beschlüssen und Julirevolution zum Prinzip der konstitutionellen Monarchie verlauten. Der allen Dingen auf den Grund gehende Denker Hegel hätte sich nicht bei der Erklärung dieses epochalen Mißverhältnisses von Begriff und Wirklichkeit per Zufall, „Schmalzgesellenschaft" einerseits, abstraktem Fanatismus des "Freiheitsgesindels“ andererseits, wie er das nannte, beruhigen dürfen. Doch das Nichtverständnis geschichtlicher Praxis als eines Zufalls absichtsloser Tätigkeit reproduziert sich jetzt als die Auffassung der Restaurationsperiode in Deutschland teils als zufällige Modifikation, „querelle d'Allemand“, wie Hegel gern sagte, teils als Überhöhung des preußischen Elends gegenüber dem internationalen Niveau bürgerlicher und sogar konstitutionell-monarchischer Entwicklung in England und Frankreich. Hegel versah das Preußen der Restaurationsperiode für echte Ausführung der Französischen Revolution. Der Rest von Realismus, den diese spekulative Weihe deutscher Misere übriglieB, wandte sich auf eine Relativierung der großen Revolution als nur 
einer historischen Form des Aufbruchs aus der feudalen in die neue bürgerliche Welt. Hegels historischer Sinn, die Konsequenz seiner historischen Methode, ließen ihn in den späten Vorlesungen über die Philosophie der Weltgeschichte eine Umstrukturierung der ganzen Geschichte der Neuzeit mit der Reformation als Drehpunkt vornehmen. Aber darin war auf totale Weise nur wieder das Spekulative der Geschichts- und politischen Auffassungen Hegels reproduziert.

Hegels Ausspruch von der absichtslosen Praxis des geschichtlichen Fortschritts bezeichnet das Dilemma seines idealistischen Geschichtsverständnisses. Hegels kritische Theorie der nachrevolutionären Gesellschaft muß ebenso unbedingt, wie sie negiert, auch affirmieren, was sich gestaltet hat; sie gäbe sonst den Anspruch auf Einheit von Denken und Sein auf. Sie ist aber für Hegel nur bei sich, wenn sie theoretisch verneint oder bejaht, sie ist außer sich während der Ausführung der Kritik. Praxis, die Entfremdung des Geistes, ist nicht ihr Feld. Darin ist der kontemplative Kern bürgerlicher kritischer Theorie gegeben. Das Wechselverhältnis von Kritik und kontemplativer Akkomodation geht durch alle Schriften Hegels hindurch. Es bildet recht eigentlich den Grundzug der Hegelschen Philosophie überhaupt. Hegels Politik, seine Religionslehre, alles steht unter der Spannung von Kritik und Rechtfertigung. Wegen dieses kritisch-konservativ gedoppelten Prinzips der großen Synthese der klassisch-bürgerlichen Weltanschauung konnten sich im Ausgang der klassischen Periode, als Hegels spekulative Rechtfertigung der existierenden Wirklichkeit in Politik und Religion nicht mehr standhielt, eine progressive wie eine konservative HegelRichtung gleichermaßen auf Hegel berufen. Sie interpretierten Hegels System, konstant in gutem Glauben, entweder nach dessen kritischem oder nach dem affirmierenden Bauelement.

In der letzten Periode seines politischen Denkens mußte Hegel den versöhnenden Grundzug des philosophischen Prinzips verstärken. Je fester die Politik im Deutschen Bund stillstand und je mehr die Mainzer Untersuchungskommission das einzige sich bewegende Kritische in ihm wurde, desto vorsichtiger, unsicherer, schweigsamer mußte 
der zwischen Revolution und Restauration stehende Hegel werden. Der Philosophie wird der Ehrentitel, kritische Theorie zu sein, ausdrücklich und recht derb aberkannt. Konkrete politische Auffassung, Entfaltung des Wissens zur reichen Anschauung der Gesellschaftswelt ist Rechtfertigung der existierenden Welt, die ihre Vernunft schon immer in sich hat. Sie braucht dazu nicht die besser wissen wollende Philosophie, die kommt ohnehin zu spät. So finden wir in der Vorrede zur Rechtsphilosophie neben dem berühmten Bild von der Eule der Minerva, die erst bei einbrechender Dämmerung ihren Flug beginne, neben den harten Verdammungsurteilen gegen eine Rechtslehre und politische Theorie, die sich erfreche, belehren zu wollen, wie der Staat sein solle, die sachliche Formulierung der Methode, nach der Hegel seine politische Theorie und sein politisches Verhalten nunmehr bildete: „Ohnehin über Recht, Sittlichkeit, Staat ist die Wahrheit ebenso sehr alt, als in den öffentlichen Gesetzen, der öffentlichen Moral und Religion offen dargelegt und bekannt. Was bedarf diese Wahrheit weiter, insofern der denkende Geist sie in dieser nächsten Weise zu besitzen nicht zufrieden ist, als sie auch zu begreifen, und dem schon an sich selbst vernünftigen Inhalt auch die vernünftige Form zu gewinnen, damit er für das freie Denken gerechtfertigt erscheine ..." Wir haben hier das ebenso gedanklich klare wie wohlstilisierte politische Bekenntnis des alten Hegel, abgeleitet aus seinem philosophischen Prinzip. Mit dem gegebenen Staat, seinem Recht und seinem Glauben nicht wohl zufrieden, wende Dich an die Philosophie, um da zu erkennen, daß „alles zu nehmen ist, wie es ist", weil es ist, wie es sein muß. Die Indolenz der ,gesetzten Leute“ kehrt zu Hegel selbst zurück, freilich als eine von spekulativer Weltmethode erhobene.

Aber wie denn nun? Wie wird uns denn, wenn wir der Worte Sinn nur richtig fassen wollen? Narrt Hegel uns oder wird er vom Geist seiner abgeklärten Spekulation selbst genarrt? Die Philosophie wird gesucht, weil sonst Unzufriedenheit mit dem öffentlich Vorhandenen ist. Die Philosophie ist noch nötig, um dem denkenden Geist erst das wirkliche Leben zu rechtfertigen, da es ohne diese vor 
ihm keine Rechtfertigung fände. Das öffentlich Wirkliche gibt wohl gar für Vorschläge, wie alles sein sollte, noch genügenden Anstoß? - Die Kritik der Wirklichkeit kommt mit stummer Gebärde in der spekulativen Rechtfertigung, die Hegel vornimmt, wieder zum Vorschein. Kritische Theorie läßt sich gerade da, wo sie endgültig verschwunden sein sollte, ihres Erstgeburtsrechts nicht berauben. Weil alles nicht ist, wie es vom denkenden Geist sein soll, legen wir uns die kolossale methodische Zucht auf, die uns endlich „Hören und Sehen vergehen läßt" 1 und zur Ruhe stillen Denkens zwingt. Was dann von der Wirklichkeit, die ohne Philosophie nicht ruhig sein kann, an Festigkeit bleibt, ist nur die zufällige „besondere Gewalt", eine nicht eben „absichtliche Tätigkeit“. Der alte Hegel akzeptiert die Gewalt, auch wenn es nicht diejenige geworden ist, die er als Dreißigjähriger auf seiner Suche nach Rettung fürs Deutsche Reich erwartet hatte. So liegt Einheit des Prinzips über allem politischen Denken Hegels; mag auch der reife Mann die Gewalt nicht mehr genau besehen, nach deren Anblick er sich als Jüngling gesehnt hatte.

Die gleiche verborgene Kritik an der eben gerechtfertigten Wirklichkeit steckt doch auch in den Sätzen, mit denen das Gleichnis vom Fluge des Weisheitsvogels eingeleitet wird. Philosophie als Gedanke der Welt erscheint erst, wenn die Wirklichkeit ihren Bildungsproze $B$ vollendet hat. Daher kommt es: „Wenn die Philosophie ihr Grau in Grau malt, dann ist eine Gestalt des Lebens alt geworden ..." Also hat sie einer neuen, jungen Gestalt Platz zu machen? Die rechtfertigende Philosophie kommt so auf einmal im Gewand des Leichenbitters. Die seriöse Akkomodation der spekulativen Dialektik ist erfüllt von Ironie.

Hegel hat diese Bestimmung, da 3 Philosophie als das Wissen des Substantiellen einer Zeit ganz identisch sei mit ihrer Zeit und doch darum gerade auch über ihrer Zeit stehe, wohl bedacht. Er sagt in den philosophiehistorischen Vorlesungen darüber: „Die Philosophie steht jedoch andererseits der Form nach über ihrer Zeit, indem sie als das Denken dessen, was der substantielle Geist derselben ist,

1 Hegel, Phänomenologie, Werke, Bd. 2, Berlin 1841, S. 97 
ihn sich zum Gegenstand macht. Insofern sie im Geiste ihrer Zeit ist, ist er ihr bestimmter weltlicher Inhalt, zugleich ist sie aber als Wissen auch darüber hinaus ... Dies Wissen ist es dann, was eine neue Form der Entwicklung hervorbringt, die neuen Formen sind nur Weisen des Wissens ... Die Philosophie ist also schon ein weiterer Charakter des Geistes, sie ist die innere Geburtsstätte des Geistes, der später zu wirklicher Gestaltung hervortreten wird." 1 Die Größe eines Denkers liegt in seiner Konsequenz. Philosophie hat hier zwar wieder den Auftrag kritischer Verarbeitung des Seienden; die kritische Funktion ist aber ausdrücklich auf theoretische Kritik beschränkt. Die ,,weitere Entwicklung", die Hegel im Auge hat, ist nur die der weiteren Philosophie. Das Problem der Realveränderung durch Vernunft und Praxis wird nicht aufgenommen. „Die neuen Formen sind nur Weisen des Wissens", und ein RealprozeB, in dem die "neuen Formen der Entwicklung“ hervortreten, kommt in der Theorie nicht vor.

Der Schacht des Irrationalen, in dem der Vernunftidealismus das Hauptwort Praxis verschließt, muB von Hegel in seinen Vorlesungen über die Weltgeschichte wieder geöffnet werden, denn hier ging es sichtlich nicht nur um neue Philosophien. Den Abgrund des Irrationalen verdecken nun die welthistorischen Individuen. Sie handeln, gewaltsam, in "nicht absichtlicher Tätigkeit" hinsichtlich des Eigentlichen ihrer Wirkungen, das in der Idee für sich bleibt, und holen mit bodenloser Inspiration dem Weltgeist, der es wissen muß, die Kastanien aus dem Feuer. So erstirbt Realpraxis überall vor dem Geheimnis der Idee. Die wirklichen kritischen Ideen der wirklichen Individuen haben damit nichts zu schaffen. Kritische Theorie bleibt theoretische Kritik, und Philosophie ist nicht von dieser Welt.

\section{JII. Marxismus-Leninismus. Die wirkliche Revolution}

Hegels politische Theorie führte auf eine Gewalt, die seine Philosophie nicht begreifen konnte. In Anerkenntnis, da $B$

1 Hegel, Werke, Bd. 13, Berlin 1833, S. 69 f. 